\title{
List of Zooplankton Taxa in the Caspian Sea Waters of Iran
}

\author{
Siamak Bagheri, ${ }^{1}$ Jalil Sabkara, ${ }^{1}$ Alireza Mirzajani, ${ }^{1,2}$ Seyed Hojat Khodaparast, ${ }^{1}$ \\ Esmaeil Yosefzad, ${ }^{1}$ and Foong Swee Yeok ${ }^{3}$ \\ ${ }^{1}$ Inland Waters Aquaculture Institute, Iranian Fisheries Research Organization (IFRO), Anzali 66, Iran \\ ${ }^{2}$ Faculty of Natural Resources, University of Tehran, P.O. Box 4314, Karaj 31587-77878, Iran \\ ${ }^{3}$ School of Biological Sciences, Universiti Sains Malaysia, 11800 Penang, Malaysia \\ Correspondence should be addressed to Siamak Bagheri; siamakbp@gmail.com
}

Received 26 June 2012; Revised 15 September 2012; Accepted 14 October 2012

Academic Editor: E. A. Pakhomov

Copyright (C) 2013 Siamak Bagheri et al. This is an open access article distributed under the Creative Commons Attribution License, which permits unrestricted use, distribution, and reproduction in any medium, provided the original work is properly cited.

\begin{abstract}
A total of 61 zooplankton taxa were found in the southwestern Caspian Sea between 1996 and 2010. Thirteen of them were meroplankton taxa and forty-eight were holoplankton taxa. The occurrence of 14 freshwater taxa indicated the influence of the Anzali wetland and river inflows. The decrease in zooplankton taxa was detected since 1996-1997 and continued till 2010. Pleopis polyphemoides, the only one out of the nine recorded Cladocera species in 1996-1997, was found after 2001. Similarly, of the five Copepoda species recorded in 1996-1997, only one, Acartia tonsa, was found abundant during the 2001-2010 sampling period. It was striking that many species which were abundant in the Caspian Sea in 1996-1997 were not found after 2000. Many reasons could have contributed to the changes in the zooplankton composition of the southern Caspian Sea, notably the serious environmental degradation since the early 1990s. It is also possible that invasive species might play a role in wiping out some sensitive endemic species.
\end{abstract}

\section{Introduction}

The Caspian Sea is a large inland water body. It is called a sea even though not being directly connected to any marine system due to its large size [1]. Water is mainly sourced from the big river Volga in the north (almost 76.3\% of the total), and other rivers like Kura, Ural, Terek, and Sefidrood [2]. In the Iranian coast, the Sefidrood river is the largest river pouring into the Caspian Sea with $67,000 \mathrm{~km}^{2}$ of catchment area and an average discharge of 4,037 million $\mathrm{m}^{3}$ per year $[3,4]$. The Caspian Sea has undergone significant ecological alteration during the past 30 years. This could be linked to dramatic changes in the southern Caspian Sea due to increased pollution: sewage, industry effluent, and agricultural waste water discharges into the river as well as deforestation of the river's watersheds $[5,6]$.

Zooplankton is recognized among the best indicators to be particularly useful to investigate and document environmental changes [7]. Main zooplankton taxa have short life cycle and the community structure is able to reflect real-time scenario as it is less enforced by the stability of individuals from previous years [8]. Besides this, zooplankton is also the food of choice for many fishes and as such plays a very significant role in pilaring the upper stages of the food chain [9].

Bagirov [10] reported that the number of zooplankton taxa was almost 200 in the northern Caspian Sea with 70 taxa of Protista, 50 taxa of Rotatoria, 30 taxa of Cladocera, and 20 taxa of Copepoda. Meroplankton, represented mainly by larvae of bivalves and crustaceans, contributed to the biodiversity of plankton communities in the North. Whereas in the South of Caspian Sea, Hossieni et al. [11] documented that zooplankton community consisted of 36 taxa, including Cladocera (24 taxa), Copepoda (7 taxa), and meroplankton (2 taxa) along the Iranian coastal area of the Caspian Sea in 1996. Subsequently in 1999, Sabkara et al. [12] reported over 50 zooplankton taxa with holoplankton comprising $>80 \%$ of the taxa sampled and Rotifera (22 species) dominating. Meroplankton accounted for the remaining diversity. Recently, Roohi et al. [13] noted that only 18 zooplankton taxa were observed in the southern Caspian Sea, of which five taxa belonged to holoplankton (four Copepoda and one Cladocera) and 13 to meroplankton. 
TABLE 1: " $\times$ ” denotes sampling done based on season in the Caspian Sea waters of Iran during 1996-2010.

\begin{tabular}{ccccc}
\hline Year & Winter & Spring & Season & \\
\hline 1996 & $\times$ & $\times$ & Summer & \\
1997 & $\times$ & & $\times$ & \\
2001 & $\times$ & & $\times$ & $\times$ \\
2002 & $\times$ & $\times$ & $\times$ & $\times$ \\
2003 & $\times$ & $\times$ & $\times$ & $\times$ \\
2004 & $\times$ & $\times$ & $\times$ & $\times$ \\
2005 & $\times$ & & $\times$ & $\times$ \\
2006 & $\times$ & $\times$ & $\times$ \\
2008 & $\times$ & $\times$ & $\times$ \\
2009 & $\times$ & & $\times$ \\
\end{tabular}

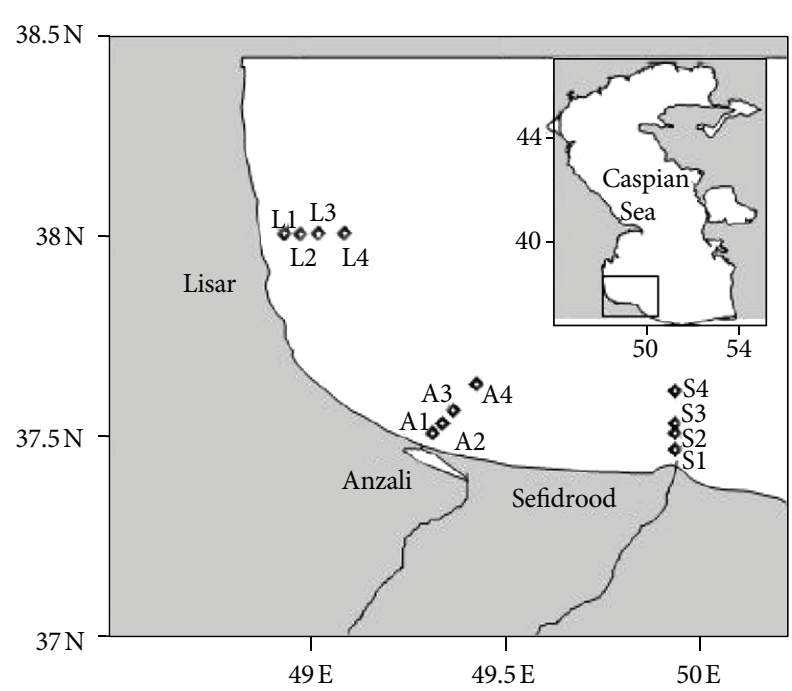

FIgURE 1: Study sites sampled 1996 to 2010 in the Caspian Sea waters of Iran. $\mathrm{L}=$ Lisar, $\mathrm{A}=$ Anzali, $\mathrm{S}=$ Sefidrood, $5 \mathrm{~m}(\mathrm{~L} 1, \mathrm{~A} 1, \mathrm{~S} 1), 10 \mathrm{~m}$ (L2, A2, S2), $20 \mathrm{~m}(\mathrm{~L} 3, \mathrm{~A} 3, \mathrm{~S} 3)$, and $50 \mathrm{~m}(\mathrm{~L} 4, \mathrm{~A} 4, \mathrm{~S} 4)$.

In recent years, few studies have been conducted on the distribution and abundance of zooplankton in the southern Caspian Sea [11-14], while there was hardly any work done to look at the composition of zooplankton taxa. In order to investigate the state of ecological condition of the Caspian Sea, an eleven-year study of the zooplankton community in the Caspian Sea waters of Iran was compiled between 1996 and 2010 .

\section{Materials and Methods}

2.1. Study Area. The area under investigation is located at the southwestern corner of the Caspian Sea. The identification of zooplankton taxa was carried out along three transects along the mouth of the Anzali wetland, Lisar and Sefidrood rivers. The sampling was performed at $5 \mathrm{~m}, 10 \mathrm{~m}, 20 \mathrm{~m}$, and $50 \mathrm{~m}$ depths during 1996-2010 (Figure 1). Table 1 shows the seasonal sample collection done in between 1996-2010. In some years, sampling was unable to be carried out due to logistic problems. In total, a collection of 132 samples were carried out during these periods (Table 1).

2.2. Data Collection. Zooplankton was sampled using a Juday net (opening diameter: $36 \mathrm{~cm}$, mesh size: $100 \mu \mathrm{m}$ ). At every station, a vertical haul with a Juday net was carried out from bottom to surface using a handle pulley for heaving the net. Zooplankton samples were preserved in neutral $4 \%$ formaldehyde and analyzed in the laboratory. Samples were divided into subsamples using a $1 \mathrm{~mL}$ Hensen-Stempel pipette and transferred to a Bogorov chamber for identification. At least 100 individuals were counted per sample and identified to species, and life-cycle stages were determined using an inverted microscope [15]. Zooplankton taxonomic classification was performed based on Birshtain et al. [16], Kasimov [17], and James and Covich [18].

\section{Results}

A total of 61 zooplankton taxa belonging to 48 taxa of holozooplankton were found in the area of investigation (Tables 2 and 3). Fourteen of them, Pleroxus trigenellus O.F. Mueller, Chydorus sp. Leach, Moina sp. Baird, all Cladocera, the copepod Cyclops sp. Risso, Cyclops sp. nauplii and the rotifers Filinia sp. Vincent, Keratella cochlearis, Lecane sp. Nitzsch, Notholca acuminate Ehrenberg, Philodina sp. Ehrenberg, Polyarthera dolichoptera Idelson, Testudinella patina Hermann, Trichocerca sp. Lamarck, and Trichocerca caspia Tschugunoff are freshwater species in the area.

Thirteen meroplankton taxa consisted of individuals of water spiders (Arachnida), crab (Rhithropanopeus harrisii Gould), Foraminifera, Ostracoda larvae, Asteromeyenia sp. Weltner, Pseudocuma sp. G.O. Sars, and larvae of Bivalvia, Nematoda, Polychaeta (Hediste diversicolor O.F. Muller, Hypania sp. Ostroumouw), Pisces (egg and larvae), and multitudinous Cirripedia (Balanus sp. Costa) represented by nauplii and cypris larvae (Table 2 ).

Thirty-four holoplankton taxa belong to Ctenophora (Mnemiopsis leidyi A. Agassiz), Ciliata (Codonella sp. Haeckel, Codonella relicta Minikiewich, Tintinnopsis sp. Lamarck, 
TABLE 2: Checklist of zooplankton taxa in the Caspian Sea waters of Iran during 1996-2010.

\begin{tabular}{|c|c|c|c|c|c|c|c|c|c|}
\hline \multirow{2}{*}{ Holoplankton taxa } & \multicolumn{9}{|c|}{ After appearance of Mnemiopsis leidyi } \\
\hline & 1996-1997 & 2001 & 2002 & 2003 & 2004 & 2005 & 2006 & 2008 & $2009-2010$ \\
\hline \multicolumn{10}{|l|}{ Macrozooplankton } \\
\hline \multicolumn{10}{|l|}{ Ctenophora: } \\
\hline${ }^{*}$ Mnemiopsis leidyi A. Agassiz, 1865 & - & + & + & + & + & + & + & + & + \\
\hline \multicolumn{10}{|l|}{ Mesozooplankton } \\
\hline \multicolumn{10}{|l|}{ Cladocera: } \\
\hline Cercopagis pengoi Ostroumov, 1891 & + & - & - & - & - & - & - & - & - \\
\hline Cercopagis prolongata G.O. Sara, 1897 & + & - & - & - & - & - & - & - & - \\
\hline Chydorus Leach, 1843 & - & - & - & - & - & - & - & + & - \\
\hline Evaden anonyx G.O. Sars, 1897 & + & - & - & - & - & - & - & - & - \\
\hline Moina Baird, 1850 & - & - & - & - & - & - & - & + & - \\
\hline Pleroxus trigonellus O.F. Muller, 1785 & - & - & - & - & - & - & - & + & - \\
\hline Podonevadne Gibitz, 1922 & - & - & - & - & - & - & - & + & - \\
\hline Podonevaden angusta G.O. Sars, 1902 & + & - & - & - & - & - & - & - & - \\
\hline Podonevaden camptonyx G.O. Sars, 1897 & + & - & - & - & - & - & - & - & - \\
\hline Podonevaden trigona G.O. Sars, 1897 & + & - & - & - & - & - & - & - & - \\
\hline Podon intermedius Lilljeborg, 1853 & + & - & - & - & - & - & - & - & - \\
\hline *Pleopis polyphemoides Leuckart, 1859 & + & - & + & - & + & + & + & + & + \\
\hline Polyphemus exiguus G.O. Sars, 1897 & + & - & - & - & - & - & - & - & - \\
\hline \multicolumn{10}{|l|}{ Copepoda: } \\
\hline${ }^{*}$ Acartia tonsa nauplii & + & + & + & + & + & + & + & + & + \\
\hline${ }^{*}$ Acartia tonsa Dana, 1849 & + & + & + & + & + & + & + & + & + \\
\hline${ }^{*}$ Calanipeda aquae dulcis nauplii & + & - & - & - & - & - & - & - & - \\
\hline${ }^{*}$ Calanipeda aquae dulcis Kritchagin, 1873 & + & - & - & - & - & - & - & - & - \\
\hline Cyclops nauplii & - & - & - & - & - & - & - & + & - \\
\hline Cyclops Risso, 1826 & + & + & - & + & + & + & - & + & + \\
\hline Eurytemora grimmi nauplii & + & - & - & - & - & - & - & - & - \\
\hline Eurytemora grimmi G.O. Sars, 1897 & + & - & - & - & - & - & - & - & - \\
\hline Halicyclops sarsi Akatova, 1935 & + & - & + & - & - & - & - & - & + \\
\hline Ectinosoma concinnum Akatova, 1935 & + & - & - & - & - & - & - & + & - \\
\hline Limnocalanus grimaldii grimaldii nauplii & + & - & - & - & - & - & - & - & - \\
\hline Limnocalanus grimaldii grimaldii Guerne, 1886 & + & - & - & - & - & - & - & - & - \\
\hline \multicolumn{10}{|l|}{ Rotifera: } \\
\hline Brachionus Pallas, 1766 & - & - & - & - & + & + & - & - & - \\
\hline Brachionus angularis Gosse, 1851 & - & + & + & - & - & - & - & - & - \\
\hline Brachionus calyciflorus Pallas, 1766 & - & - & + & - & + & + & + & - & + \\
\hline Brachionus plicatilis Muller, 1786 & - & + & + & - & - & - & - & + & + \\
\hline Filinia Bory de St. Vincent, 1824 & - & - & + & - & - & - & - & - & - \\
\hline Keratella cochlearis Gosse, 1851 & - & + & + & + & - & - & - & + & + \\
\hline Lecane Nitzsch, 1827 & - & + & - & - & - & - & - & - & - \\
\hline Notholca acuminata Ehrenberg, 1832 & - & - & + & - & - & - & - & - & - \\
\hline Philodina Ehrenberg, 1830 & - & - & - & - & - & - & - & - & + \\
\hline Polyarthera dolichoptera Idelson, 1925 & - & - & + & - & - & - & - & - & + \\
\hline Synchaeta Ehrenberg, 1832 & + & + & + & + & + & + & + & - & + \\
\hline Synchaeta stylata Wierzejski, 1893 & + & - & - & - & - & - & - & - & - \\
\hline Synchaeta vorax Rousselet, 1902 & + & - & - & - & - & - & + & - & - \\
\hline Testudinella patina Hermann, 1783 & - & - & - & - & - & - & - & - & + \\
\hline Trichocerca Lamarck, 1801 & - & - & + & - & - & - & - & - & - \\
\hline Trichocerca capica Tschugunoff, 1921 & - & - & + & - & - & - & - & - & - \\
\hline \multicolumn{10}{|l|}{ Microzooplankton } \\
\hline \multicolumn{10}{|l|}{ Ciliata: } \\
\hline Codonella Haeckel, 1873 & - & - & + & - & - & - & - & - & - \\
\hline
\end{tabular}


TABLE 2: Continued.

\begin{tabular}{|c|c|c|c|c|c|c|c|c|c|}
\hline \multirow{2}{*}{ Holoplankton taxa } & \multicolumn{9}{|c|}{ After appearance of Mnemiopsis leidyi } \\
\hline & 1996-1997 & 2001 & 2002 & 2003 & 2004 & 2005 & 2006 & 2008 & $2009-2010$ \\
\hline Codonella relicta Minikiewich, 1905 & - & + & + & - & - & - & - & - & + \\
\hline Tintinnopsis Stein 1867 & - & + & + & - & - & - & - & - & - \\
\hline Tintinnopsis karajacensis Brandt, 1896 & - & + & - & - & - & - & - & - & - \\
\hline Tintinnopsis tubulosa Levander, 1900 & - & + & + & + & + & + & - & + & + \\
\hline \multicolumn{10}{|l|}{ Meroplankton taxa (Mesozooplankton) } \\
\hline Arachnida Larvae Cuvier, 1812 & + & - & - & - & - & - & - & + & + \\
\hline \multicolumn{10}{|l|}{ Cirripedia: } \\
\hline${ }^{*}$ Balanus sp. cypris Costa, 1778 & + & + & + & + & + & + & + & + & + \\
\hline${ }^{*}$ Balanus sp. nauplii Costa, 1778 & + & + & + & + & + & + & + & + & + \\
\hline \multicolumn{10}{|l|}{ Crustacea: } \\
\hline${ }^{*}$ Rhithropanopeus harrisii Gould, 1841 & + & - & - & - & - & - & - & - & - \\
\hline Foraminifera larvae & + & + & + & - & + & + & - & - & + \\
\hline Bivalvia larvae Linnaeus, 1758 & + & + & + & + & + & + & + & + & - \\
\hline Nematoda larvae & - & - & - & - & + & + & + & + & + \\
\hline Ostracoda larvae Latreille, 1802 & - & - & + & - & - & - & - & - & - \\
\hline Pisces larvae & + & + & + & - & + & + & + & + & + \\
\hline Pisces ovae & + & + & - & - & + & + & + & + & + \\
\hline \multicolumn{10}{|l|}{ Polychaeta: } \\
\hline Hypania Ostroumouw, 1897 & - & - & - & - & - & - & - & + & - \\
\hline Hediste diversicolor O.F. Muller, 1776 & - & + & + & + & + & + & + & + & + \\
\hline \multicolumn{10}{|l|}{ Porifera: } \\
\hline Asteromeyenia Weltner, 1913 & - & - & - & - & - & - & - & + & - \\
\hline \multicolumn{10}{|l|}{ Cumacea: } \\
\hline Pseudocuma G.O. Sars, 1865 & + & - & - & - & - & - & - & - & - \\
\hline
\end{tabular}

Alien taxa; bold font: freshwater taxa indicated the influence by river inflow.

TABle 3: Zooplankton number of taxa (holomeroplankton) in the Caspian Sea waters of Iran during 1996-2010.

\begin{tabular}{|c|c|c|c|c|c|c|c|c|c|c|}
\hline \multirow{2}{*}{ Zooplankton taxa } & \multicolumn{9}{|c|}{ After appearance of Mnemiopsis leidyi } & \multirow{2}{*}{ Number of taxa } \\
\hline & 1996-1997 & 2001 & 2002 & 2003 & 2004 & 2005 & 2006 & 2008 & 2009-2010 & \\
\hline Ctenophora & 0 & 1 & 1 & 1 & 1 & 1 & 1 & 1 & 1 & 1 \\
\hline Ciliata & 0 & 4 & 4 & 1 & 1 & 1 & 0 & 1 & 2 & 5 \\
\hline Cladocera & 9 & 0 & 1 & 0 & 1 & 1 & 1 & 5 & 1 & 13 \\
\hline Copepoda & 11 & 3 & 3 & 3 & 3 & 3 & 2 & 5 & 4 & 12 \\
\hline Rotifera & 3 & 5 & 10 & 2 & 3 & 3 & 3 & 2 & 7 & 17 \\
\hline Total holoplankton & 23 & 13 & 19 & 7 & 9 & 9 & 7 & 14 & 15 & 48 \\
\hline Arachnida & 1 & 0 & 0 & 0 & 0 & 0 & 0 & 1 & 1 & 1 \\
\hline Cirripedia & 2 & 2 & 2 & 2 & 2 & 2 & 2 & 2 & 2 & 2 \\
\hline Crustacea & 1 & 0 & 0 & 0 & 0 & 0 & 0 & 0 & 0 & 1 \\
\hline Foraminifera & 1 & 1 & 1 & 0 & 1 & 1 & 0 & 0 & 1 & 1 \\
\hline Bivalvia & 1 & 1 & 1 & 1 & 1 & 1 & 1 & 1 & 0 & 1 \\
\hline Nematoda & 0 & 0 & 0 & 0 & 1 & 1 & 1 & 1 & 1 & 1 \\
\hline Ostracoda & 0 & 0 & 1 & 0 & 0 & 0 & 0 & 0 & 0 & 1 \\
\hline Pisces & 2 & 2 & 1 & 0 & 2 & 2 & 2 & 2 & 2 & 2 \\
\hline Polychaeta & 0 & 1 & 1 & 1 & 1 & 1 & 1 & 2 & 1 & 1 \\
\hline Porifera & 0 & 0 & 0 & 0 & 0 & 0 & 0 & 1 & 0 & 1 \\
\hline Pseudocuma & 1 & 0 & 0 & 0 & 0 & 0 & 0 & 0 & 0 & 1 \\
\hline Total meroplankton & 9 & 7 & 7 & 4 & 8 & 8 & 7 & 10 & 8 & 13 \\
\hline Total zooplankton & 32 & 20 & 26 & 11 & 17 & 17 & 14 & 24 & 23 & 61 \\
\hline
\end{tabular}


TABLE 4: Seasonal list of zooplankton taxa in the Caspian Sea waters of Iran during 1996-2010.

\begin{tabular}{|c|c|c|c|c|c|}
\hline \multirow{2}{*}{ Group } & \multirow{2}{*}{ Zooplankton taxa } & \multicolumn{4}{|c|}{ Season } \\
\hline & & Winter & Spring & Summer & Autumn \\
\hline Arachnida & Arachnida larvae & + & - & - & + \\
\hline Bivalvia & Bivalvia larvae & + & + & + & + \\
\hline Ciliata & Codonella sp. & + & - & - & - \\
\hline Ciliata & Codonella relicta & + & - & - & - \\
\hline Ciliata & Tintinnopsis sp. & + & - & - & - \\
\hline Ciliata & Tintinnopsis karajacensis & + & - & - & + \\
\hline Ciliata & Tintinnopsis tubulosa & + & - & + & + \\
\hline Cirripedia & Balanus sp. cypris & + & + & + & + \\
\hline Cirripedia & Balanus sp. nauplii & + & + & + & + \\
\hline Cladocera & Cercopagis pengoi & - & - & - & + \\
\hline Cladocera & Cercopagis prolongata & - & - & - & + \\
\hline Cladocera & Chydorus sp. & + & - & - & - \\
\hline Cladocera & Evaden anonyx & - & + & - & + \\
\hline Cladocera & Moina sp. & - & - & - & + \\
\hline Cladocera & Pleroxus trigonellus & - & - & - & + \\
\hline Cladocera & Podonevadne sp. & - & + & - & - \\
\hline Cladocera & Podonevaden angusta & + & - & - & + \\
\hline Cladocera & Podonevaden camptonyx & - & + & - & - \\
\hline Cladocera & Podonevaden trigona & + & + & + & + \\
\hline Cladocera & Podon intermedius & + & - & - & - \\
\hline Cladocera & Pleopis polyphemoides & + & + & - & + \\
\hline Cladocera & Polyphemus exiguus & + & + & + & + \\
\hline Copepoda & Acartia tonsa nauplii & + & + & + & + \\
\hline Copepoda & Acartia tonsa & + & + & + & + \\
\hline Copepoda & Calanipeda aquae dulcis nauplii & + & + & + & + \\
\hline Copepoda & Calanipeda aquae dulcis & + & + & + & + \\
\hline Copepoda & Cyclops nauplii & + & - & - & + \\
\hline Copepoda & Cyclops sp. & + & - & - & + \\
\hline Copepoda & Eurytemora grimmi nauplii & + & + & + & + \\
\hline Copepoda & Eurytemora grimmi & + & + & + & + \\
\hline Copepoda & Halicyclops sarsi & + & + & + & + \\
\hline Copepoda & Ectinosoma concinnum & + & + & + & + \\
\hline Copepoda & Limnocalanus grimaldii grimaldii nauplii & + & + & + & + \\
\hline Copepoda & Limnocalanus grimaldii grimaldii & + & + & + & + \\
\hline Ctenophora & Mnemiopsis leidyi & + & + & + & + \\
\hline Crustacea & Rhithropanopeus harrisii & + & - & - & + \\
\hline Cumacea & Pseudocuma sp. & - & - & - & + \\
\hline Foraminifera & Foraminifera larvae & - & + & + & - \\
\hline Nematoda & Nematoda larvae & + & + & - & + \\
\hline Ostracoda & Ostracoda larvae & + & - & - & - \\
\hline Pisces & Pisces larvae & + & - & + & - \\
\hline Pisces & Pisces ovae & + & + & - & - \\
\hline Polychaeta & Hypania sp. & - & - & + & + \\
\hline Polychaeta & Hediste diversicolor & + & + & + & + \\
\hline Porifera & Asteromeyenia sp. & - & + & - & + \\
\hline Rotifera & Brachionus sp. & + & + & - & + \\
\hline Rotifera & Brachionus angularis & + & + & - & + \\
\hline Rotifera & Brachionus calyciflorus & + & + & - & + \\
\hline Rotifera & Brachionus plicatilis & + & + & - & + \\
\hline Rotifera & Filinia sp. & + & - & - & - \\
\hline Rotifera & Keratella cochlearis & + & - & - & - \\
\hline Rotifera & Lecane sp. & + & - & - & - \\
\hline
\end{tabular}


TABle 4: Continued.

\begin{tabular}{|c|c|c|c|c|c|}
\hline \multirow{2}{*}{ Group } & \multirow{2}{*}{ Zooplankton taxa } & \multicolumn{4}{|c|}{ Season } \\
\hline & & Winter & Spring & Summer & Autumn \\
\hline Rotifera & Notholca acuminata & + & - & - & - \\
\hline Rotifera & Philodina sp. & - & + & - & - \\
\hline Rotifera & Polyarthera dolichoptera & - & + & - & - \\
\hline Rotifera & Synchaeta sp. & + & + & - & - \\
\hline Rotifera & Synchaeta stylata & + & + & - & + \\
\hline Rotifera & Synchaeta vorax & + & + & - & + \\
\hline Rotifera & Testudinella patina & - & - & - & + \\
\hline Rotifera & Trichocerca sp. & + & + & - & - \\
\hline Rotifera & Trichocerca capica & + & - & - & - \\
\hline
\end{tabular}

Bold font: freshwater taxa indicated the influence by river inflow.

Tintinnopsis karajacensis Brandt, and Tintinnopsis tubulosa Levander), Rotifera (Brachionus sp. Pallas, Brachionus angularis Gosse, Brachionus calyciflorus Pallas, Brachionus plicatilis Mueller, Synchaeta sp. Ehrenberg, Synchaeta stylata Wierzejski, and Synchaeta vorax Rousselet), Cladocera (Cercopagis pengoi Ostroumov, Cercopagis prolongata G.O. Sars, Evaden anonyx Sars, Podonevadne sp. Gibitz, Podonevaden angusta G.O. Sars, Podonevaden camptonyx G.O. Sars, Podonevaden trigona G.O. Sars, Podon intermedius Lilljeborg, Pleopis polyphemoides Leuckart, and Polyphemus exiguus G.O. Sars), and Copepoda (Acartia tonsa Dana, Calanipeda aquae dulcis Kritchagin, Eurytemora grimmi G.O. Sars, Halicyclops sarsi Akatova, Ectinosoma concinnum Akatova, and Limnocalanus grimaldii grimaldii Guerne).

The Copepoda (Eurytemora grimmi, Limnocalanus grimaldii grimaldii, Acartia tonsa, Ectinosoma concinnum, and Halicyclops sarsi), Cladocera (Podonevaden trigona, Pleopis polyphemoides, Polyphemos exiguus), Cirripedia (Balanus sp.), Polychaeta (Hediste diversicolor), Bivalvia larvae, and Ctenophora (Mnemiopsis leidyi) dominated the zooplankton taxa in all seasons in the southwestern Caspian Sea (Table 4).

\section{Discussion}

In comparison with earlier surveys by Hossieni et al. [11] and Roohi et al. [13] which were carried out in the southern Caspian Sea, major changes in zooplankton community became obvious after 2000 (Tables 2 and 3). During the present study, 26 taxa including Codonella sp. and Codonella relicta (Ciliata); Tintinnopsis sp., Tintinnopsis karajacensis, and Tintinnopsis tubulosa (Ciliata); Brachionus sp., Brachionus angularis, Brachionus calyciflorus, and Brachionus plicatilis (Rotifera); Filinia sp. (Rotifera), Keratella cochlearis (Rotifera), Lecane (Rotifera), Notholca acuminata (Rotifera), Philodina (Rotifera), Polyarthera dolichoptera (Rotifera), Synchaeta sp. (Rotifera), Synchaeta stylata, Synchaeta vorax (Rotifera), Testudinella patina (Rotifera), Trichocerca (Rotifera), and Trichocerca capica; Podonevadne sp. (Cladocera); Rhithropanopeus harrisii (Crustacea); Asteromeyenia sp. (Porifera); Pseudocuma sp. (Cumacea) and Hypania larvae (Polychaeta) were documented. Hossieni et al. [11] and Roohi et al. [13] listed none of these taxa.
Hossieni et al. [11] did not list the following species, which were sampled in subsequent years: Ostracoda larvae, Arachnida larvae, Harpacticoida (Ectinosoma concinnum), Nematoda larvae, and Hediste diversicolor. Roohi et al. [13] listed Chironomidae, Nematidae, and Mysidacaea $(n=2$ in 2006), which were not observed in this study.

Hossieni et al. [11] listed 24 Cladocera species in the southern Caspian Sea (cited by Roohi et al., [13]). Three of these taxa were repeated twice under different names as Apagis cylindrata (Cercopagis cylindrata), Apagis longicaudata (Cercopagis longicaudata), and Apagis ossiani (Cercopagis ossiani). Additionally fourteen taxa cited were not recognized as validly published taxa by CSBP (Caspian Sea Biodiversity Project), ITIS (Integrated Taxonomic Information System), MarBEF (Marine Biodiversity and Ecosystem Functioning), and WoRNS (World Register of Marine Species), while there were nine Cladocera listed in the present study in 1996-1997 and only two of them (Pleopis polyphemoides and Podonevadne sp.) could be found after 2000. Five Copepoda species were present in 1996 [11] and the present study, but they were either absent or found in very low numbers during 2001 to 2010 (Table 2): Calanipeda aquae dulcis, Limnocalanus grimaldii, Eurytemora minor, and Eurytemora grimmi.

It was striking that many species which were abundant in the Caspian Sea since 1996-1997 were not found after 2000 (Tables 3 and 4). However, a similar drastic decline in species numbers was not observed in other invaded seas [19]. Possible reasons could be twofold: the endemic Caspian Sea fauna is very sensitive to disruptions of invaders [5,20], or it a results of serious environmental degradation which started since the beginning of the 1990s [5]. Rodionov [21], Bilio and Niermann [22], and Polonskii et al. [23] theorized that hydrobiological changes in the Caspian Sea, Black Sea, and Baltic Sea during the 1990s and 2000s could definitely be correlated to these changes. Recent remarks on other seas indicated that the shifting plankton communities can also be related to climatic inconsistency [7, 24-26].

The survey helped to characterize the composition of the zooplankton taxa in the southwestern Caspian Sea between 1996 and 2010. In comparison with previous publications, the multiyear composition of zooplankton taxa was estimated for the first time. We call for a standardization in the result 
presentation during future studies in this area. A comprehensive database including international participation should be launched to facilitate long-term comparisons of planktonic species to monitor anthropogenic and climatic effects on the Caspian Sea ecosystem.

\section{Acknowledgments}

The authors are grateful to Peter Boyce for improving the English of the draft paper. They would like to thank the Inland Waters Aquaculture Institute and Iranian Fisheries Research Organization (IFRO) for financially supporting this project. They deeply appreciate the assistance received from F. Maddadi, Y. Zahmatkesh, M. Sayad-Rahim, A. SedaghatKish, H. Norouzi, and M. Iran-Pour in this study.

\section{References}

[1] V. A. Putans, L. R. Merklin, and O. V. Levchenko, "Sediment waves and other forms as evidence of geohazards in Caspian Sea," International Journal of Offshore and Polar Engineering, vol. 20, no. 4, pp. 241-246, 2010.

[2] H. J. Dumont, "The Caspian Lake: history, biota, structure, and function," Limnology and Oceanography, vol. 43, no. 1, pp. 44-52, 1998.

[3] H. A. Lahijani, V. Tavakoli, and A. H. Amini, "South Caspian river mouth configuration under human impact and sea level fluctuations," Environmental Sciences, vol. 5, pp. 65-86, 2008.

[4] S. Bagheri, M. Mansor, M. Turkoglu, W. O. Wan Maznah, and H. Babaei, "Temporal distribution of phytoplankton in the southwestern Caspian Sea during 2009-2010: a comparison with previous surveys," Journal of the Marine Biological Association of the United Kingdom, vol. 92, pp. 1243-1255, 2012.

[5] H. Dumont, "Ecocide in the Caspian Sea," Nature, vol. 377, no. 6551, pp. 673-674, 1995.

[6] S. Bagheri, M. Mansor, M. Marzieh et al., "Fluctuations of phyoplankton community in the coastal waters of Caspian Sea in 2006," American Journal of Applied Sciences, vol. 8, no. 12, pp. 1328-1336, 2011.

[7] C. Sipkay, K. T. Kiss, C. Vadadi-Fülöp, and L. Hufnagel, “Trends in research on the possible effects of climate change concerning aquatic ecosystems with special emphasis on the modelling approach," Applied Ecology and Environmental Research, vol. 17, no. 2, pp. 171-198, 2009.

[8] A. J. Richardson, "In hot water: zooplankton and climate change," ICES Journal of Marine Science, vol. 65, no. 3, pp. 279-295, 2008.

[9] A. H. Taylor, J. I. Allen, and P. A. Clark, "Extraction of a weak climatic signal by an ecosystem," Nature, vol. 416, no. 6881, pp. 629-632, 2002.

[10] R. M. Bagirov, The Azov and Black Sea species introduced to the Caspian benthos and biofouling [Ph.D. thesis], University of Baku, 1989.

[11] A. Hossieni, A. Roohi, K. A. Ganjian et al., "Hydrology and hydrobiology of the southern Caspian Sea," Registration 96.132, Agricultural Research and Education Organization, 1998.

[12] J. Sabkara, S. Bagheri, and M. Makaremi, "Identification of Cladocera in the Caspian Sea," Journal of Fisheries Sciences, vol. 5, pp. 61-76, 2011.
[13] A. Roohi, Z. Yasin, A. E. Kideys, A. T. S. Hwai, A. G. Khanari, and E. Eker-Develi, "Impact of a new invasive ctenophore (Mnemiopsis leidyi) on the zooplankton community of the Southern Caspian sea," Marine Ecology, vol. 29, no. 4, pp. 421-434, 2008.

[14] A. Ganjian, W. O. Wan Maznah, K. Yahya et al., "Seasonal and regional distribution of phytoplanktonin the southern part of the Caspian Sea," Iranian Journal of Fisheries Sciences, vol. 9, no. 3, pp. 382-402, 2010.

[15] R. P. Harris, P. H. Wiebe, J. Lenz, and H. R. Skjoldal, Zooplankton Methodology Manual, Academic, 2000.

[16] Y. A. Birshtain, L. G. Vinogradova, N. N. Kondakov, M. S. Koon, T. V. Astakhova, and N. N. Romanova, Invertebrate Atlas Caspian Sea, Industry Food, Moscow, Russia, 1968.

[17] A. Kasimov, Methods of Monitoring in Caspian Sea, QAPPPOLIQRAF, Azerbaijan, 2000.

[18] H. T. James and A. P. Covich, Ecology and Classification of North American Freshwater Invertebrates, Academic press, 2nd edition, 2001.

[19] J. E. Purcell, T. A. Shiganova, M. B. Decker, and E. D. Houde, "The ctenophore Mnemiopsis in native and exotic habitats: U.S. estuaries versus the Black Sea basin," Hydrobiologia, vol. 451, pp. 145-176, 2001.

[20] S. Bagheri, Ecological assessment of plankton community and effects of alien species in the southwestern Caspian Sea [Ph.D. thesis], Universiti Sains Malaysia, 2012.

[21] S. N. Rodionov, Global and Regional Climate Interaction: The Caspian Sea Experience, Kluwer Academic, Dordrecht, The Netherlands, 1994.

[22] M. Bilio and U. Niermann, "Is the comb jelly really to blame for it all? Mnemiopsis leidyi and the ecological concerns about the Caspian Sea," Marine Ecology Progress Series, vol. 269, pp. 173-183, 2004.

[23] A. B. Polonskii, D. V. Basharin, E. N. Voskresenskaya, and S. Worley, "North atlantic oscillation: description, mechanisms, and influence on the eurasian climate," Physical Oceanography, vol. 14, no. 2, pp. 96-113, 2004.

[24] S. Bagheri, M. Mansor, M. Turkoglu, M. Marzieh, W. O. Wan Maznah, and H. Negaresatan, "Phytoplankton composition and abundance in the Southwestern Caspian Sea," Ekoloji, vol. 21, pp. 32-43, 2012.

[25] U. Sommer and A. Lewandowska, "Climate change and the phytoplankton spring bloom: warming and overwintering zooplankton have similar effects on phytoplankton," Global Change Biology, vol. 17, no. 1, pp. 154-162, 2011.

[26] S. Bagheri, U. Niermann, J. Sabkara, A. Mirzajani, and H. Babaei, "State of Mnemiopsis leidyi (Ctenophora: Lobata) and mesozooplankton in Iranian waters of the Caspian Sea during 2008 in comparison with previous surveys," Iranian Journal of Fisheries Sciences, vol. 11, no. 4, pp. 732-754, 2012. 

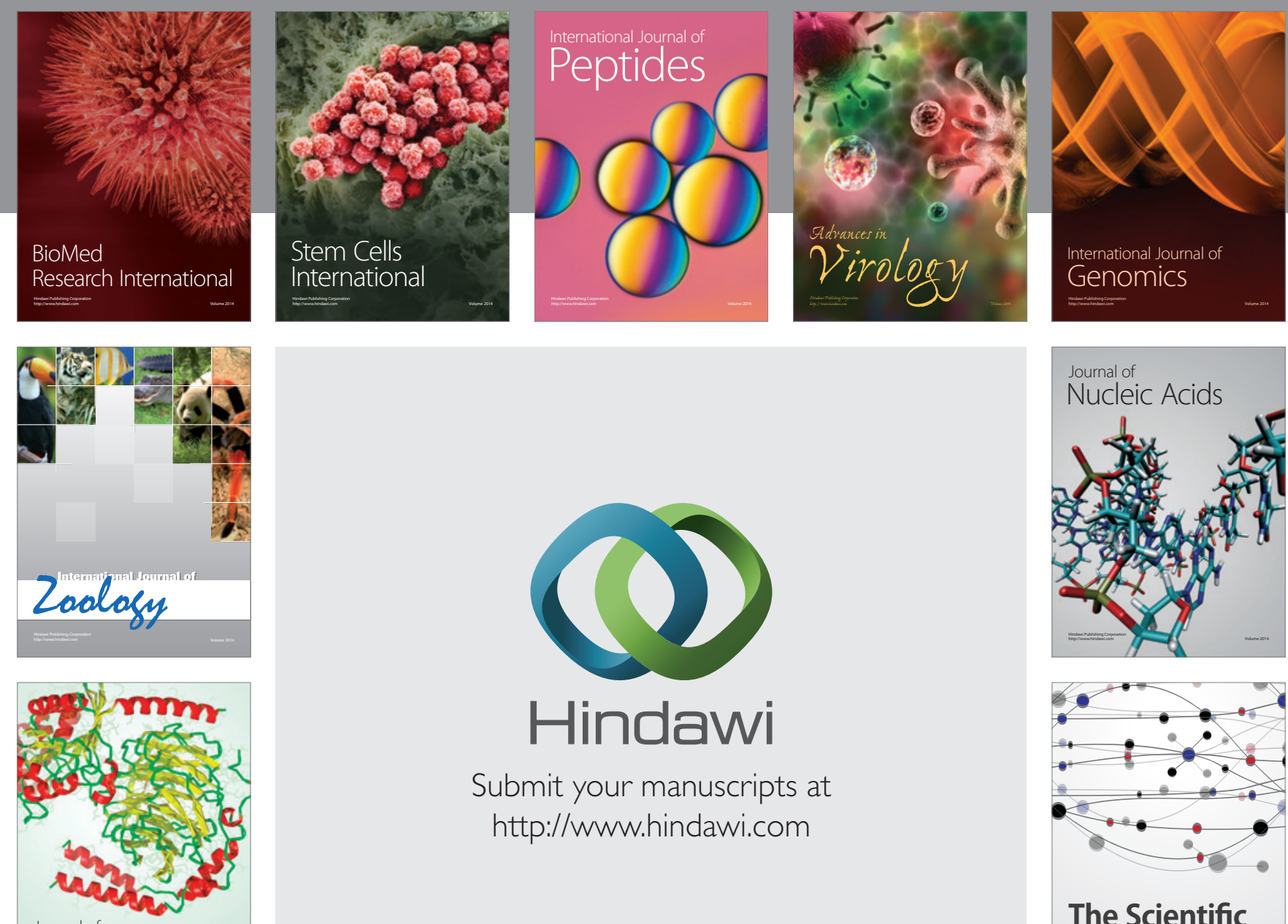

Submit your manuscripts at

http://www.hindawi.com

Journal of
Signal Transduction
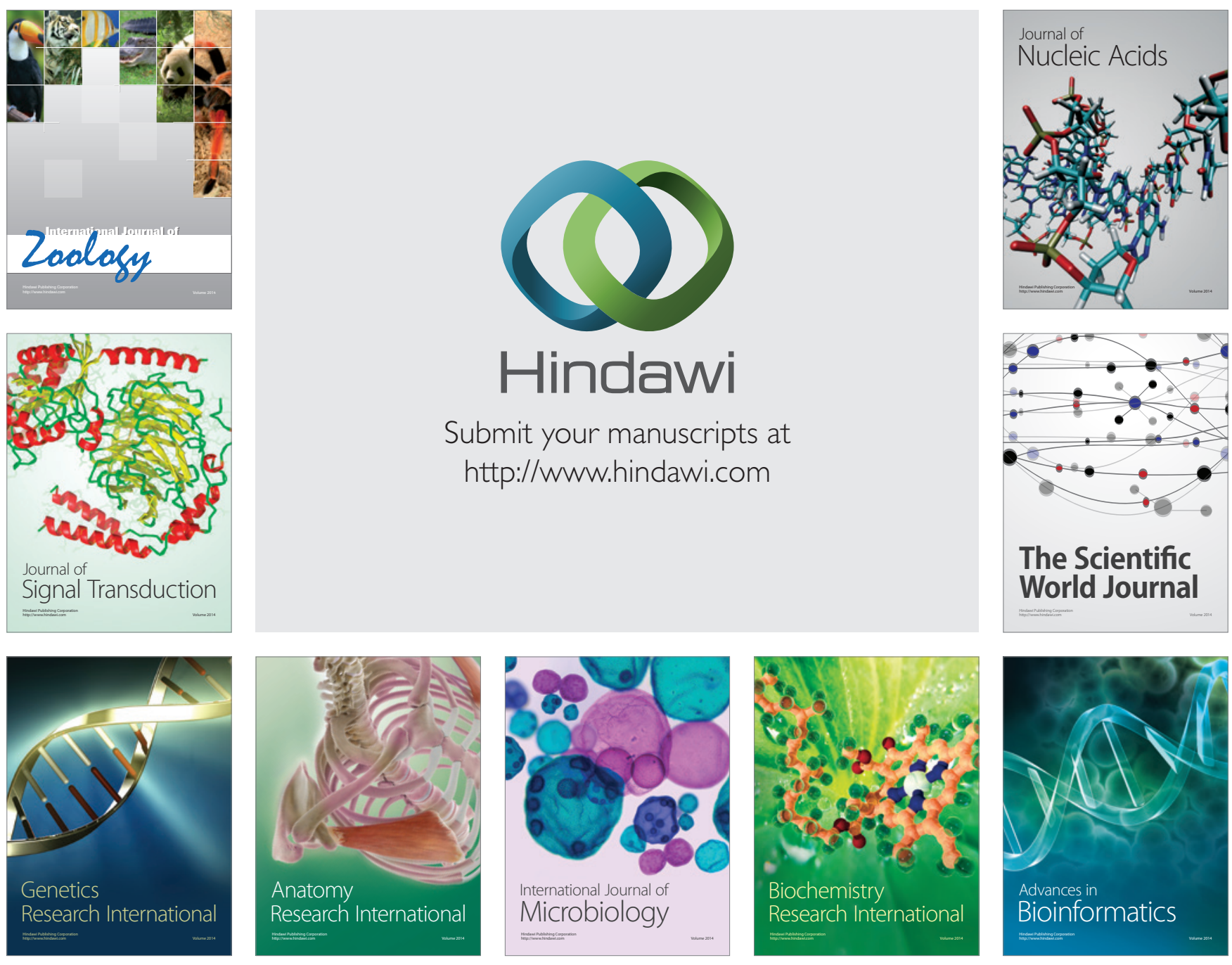

The Scientific World Journal
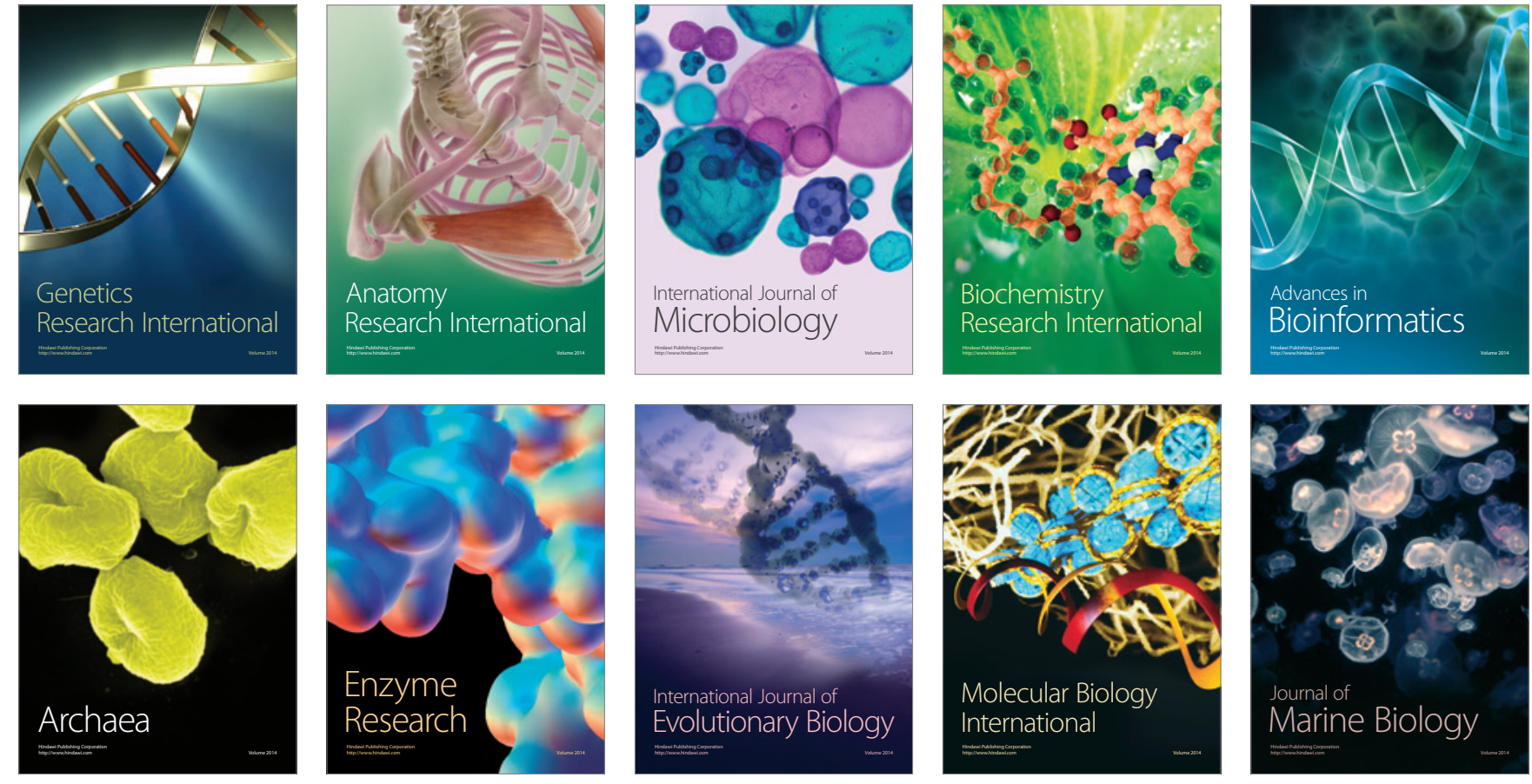